\title{
Improvement of Magnetic Properties and Texture of FePt Thin Films on MgO Substrates by Sn Addition
}

\author{
DongWon Chun ${ }^{1}$, SungMan Kim ${ }^{1}$, GyeungHo Kim ${ }^{1}$, and Won Young Jeung ${ }^{1 *}$ \\ ${ }^{1}$ Division of Materials Research, KIST, Seoul 136-791, Korea
}

(Received 7 November 2008, Received in final form 11 February 2009, Accepted 4 March 2009)

\begin{abstract}
In this work, we studied the effects of Sn addition on the ordering temperature of FePt thin film. The coercivity of FePtSn film was about 1000 Oe greater than the coercivity of $\mathrm{FePt}$ film for an annealing temperature of $600^{\circ} \mathrm{C}$. Therefore, Sn addition was effective in promoting the $L 1_{0}$ ordering and in reducing the ordering temperature of the FePt film. From our X-ray diffraction results, we found that in the as-deposited film, the addition of $\mathrm{Sn}$ induced a lattice expansion in disordered FePt thin films. After the annealing process, the excess Sn diffuses out from the ordered FePt thin film because of the difference in the solid solubility of Sn between the disordered and ordered phases. The existence of precipitates of Sn from the FePt lattice was deduced by Curie temperature measurements of the FePt and FePtSn films. Therefore, the key role played by the addition of Sn to the FePt film can be explained by a reduction in the activation energy for the $L 1_{0}$ order-disorder transformation of FePt which originates from the high internal stress in the disordered phase induced by the supersaturated Sn atoms.
\end{abstract}

Keywords : recording media, FePtSn thin film, $L 1_{0}$ ordering temperature

\section{Introduction}

$L 1_{0}$ ordered FePt alloy has attracted a great deal of attention for its applications in ultra-high-density magnetic hard disk drives because of its corrosion resistivity and its high magnetocrystalline anisotropy $\left(\mathrm{K}_{\mathrm{u}}=7 \times 10^{6}\right.$ $\mathrm{Jm}^{-3}$ ) [1-5]. However, in order to use the FePt alloy as a high-density perpendicular recording medium, it is essential both to reduce the ordering temperature and to fabricate (001)-orientated growth of $L 1_{0} \mathrm{FePt}$ thin films. In particular, the fabrication of $L 1_{0} \mathrm{FePt}$ alloy demands substrate heating during deposition or post annealing or both, and therefore one of the challenging tasks for developing these films for hard-disk-drive applications may be reducting the ordering temperature of FePt alloys. To date significant progress has been made toward reducing this ordering temperature. Stress or strain induced by under-layers, such as $\mathrm{CuRu}$ [3], $\mathrm{Ag}$ [6] and PtMn [7], has been shown to promote the ordering process of $\mathrm{FePt}$ alloys, as has the addition of third alloying elements such as $\mathrm{Cu}$ and $\mathrm{Ag}[8,9]$. A similar effect has been reported in the $L 1_{0} \mathrm{CoPt}$ alloy system, where $\mathrm{Sn}, \mathrm{Pb}, \mathrm{Sb}$, and $\mathrm{Bi}$

*Corresponding author: Tel: +82-2-958-5422

Fax: +82-2-958-6839, e-mail: wyjeung@kist.re.kr additives were shown to reduce the ordering temperature [10].

As described in this article, we attempted to reduce the order-disorder transformation temperature of Fe-Pt alloy by incorporating $\mathrm{Sn}$ as an alloying element, and we investigated the effects of that $\mathrm{Sn}$ addition. The solubility of Sn in the FePt alloy is limited. On the other hand, the high surface tension and low melting point of Sn may enhance its diffusion in the matrix even at relatively low temperatures, which may in turn lead to transient effects on the order-disorder transformation of the FePt alloy. The dissolving of excess $\mathrm{Sn}$ in disordered FePt induces lattice strain and defects, which lead to an easier transformation to an ordered $L 1_{0} \mathrm{FePt}$ alloy. Since excess $\mathrm{Sn}$ can diffuse out from the FePt lattice during the transformation process of the FePt alloy, the Sn does not have any detrimental effect on magnetic properties of $L 1_{0} \mathrm{FePt}$ alloy. In our study we examined both the magnetic properties and the microstructure modification of FePtSn to elucidate the mechanism for the ordering of the FePt alloy at low temperatures.

\section{Experimental Process}

Films made from both $\mathrm{FePt}$ and $\mathrm{FePt}$ with 1.3 at.\% $\mathrm{Sn}$ 
added (hereafter FePtSn) were deposited on $\mathrm{MgO}(100)$ single-crystal substrates using the DC and RF magnetron co-sputtering (AJA Int. Corp., ORION-8-HV) of $\mathrm{Fe}, \mathrm{Pt}$, and Sn targets with a base pressure of more than $5 \times 10^{-8}$ Torr and a sputtering Ar pressure of 3 mTorr. The substrate temperature was fixed at $150^{\circ} \mathrm{C}$ during the deposition process, and the thickness of the FePt and FePtSn films was set to $10 \mathrm{~nm}$. The post-annealing process was performed in a vacuum chamber at $4 \times 10^{-8}$ Torr and temperatures from $400-600^{\circ} \mathrm{C}$, and the annealing time was $30 \mathrm{~min}$. The crystal structures of the FePt and FePtSn thin films were characterized by an X-ray diffractometer (Bruker, D-8) using $\mathrm{Cu}-\mathrm{K \alpha}$ radiation at $40 \mathrm{kV}$ and 40 $\mathrm{mA}$, and the microstructure of the prepared films was investigated by a field-emission transmission electron microscope (FE-TEM, Tecnai F20G2) operating at 200 $\mathrm{kV}$. The magnetic properties and Curie temperatures $\left(T_{\mathrm{C}}\right)$ were measured using a vibrating sample magnetometer (VSM, Lakeshore 662) with an applied field of up to \pm 15 kOe. The Fe and Pt contents of all the films, measured by means of inductively coupled plasma atomic emission spectrometry, were found to have an almost constant ratio of 49.5:50.5.

\section{Results and Discussion}

Fig. 1 shows the out-of-plane coercivity $\left(H_{\mathrm{c}}\right)$ of the $\mathrm{FePt}$ and FePtSn films as a function of the annealing temperature. When the annealing temperature is below $400^{\circ} \mathrm{C}$, the $H_{\mathrm{c}}$ of the FePt and FePtSn films did not exceed $300 \mathrm{Oe}$, indicating that both films remained in the disordered phase. As the annealing temperature increased above $500^{\circ} \mathrm{C}$, the $H_{\mathrm{c}}$ of the FePt and FePtSn films increased rapidly, indicating that both films were experi-

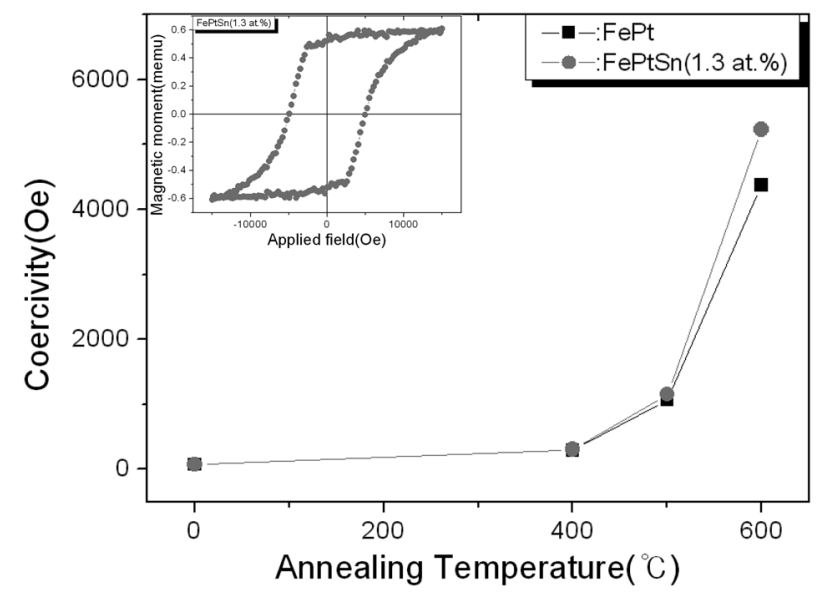

Fig. 1. Temperature dependency of the out-of-plane coercivity of the FePt and FePtSn thin films and (inset) out-of-plane hysteresis loop of FePtSn (1.3 at.\%) thin film.

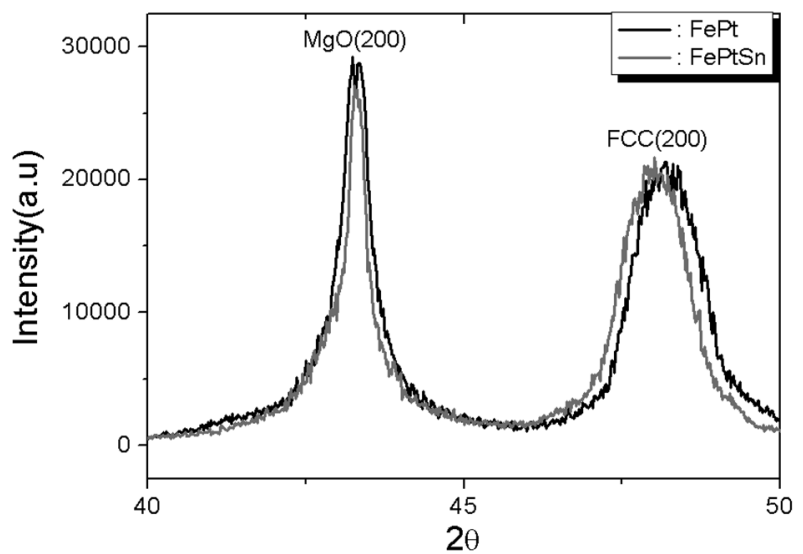

Fig. 2. X-ray diffraction of the as-deposited FePt and FePtSn thin films.

encing the phase transformation to the ordered $L 1_{0}$ phase. Of particular importance is the fact that the $H_{\mathrm{c}}$ of the FePtSn film was higher than that of the FePt film, with an enhancement of 1000 Oe achieved at the annealing temperature of $600^{\circ} \mathrm{C}$. This suggests that the addition of the $\mathrm{Sn}$ enhances the $L 1_{0}$ ordering. Our results clearly show that the addition of Sn effectively lowers the ordering temperature for the formation of the $L 1_{0}$ phase in the Fe-Pt alloy and that it increases the coercivity of the FePt film.

To investigate the effects of the Sn on the FePt film, we performed a crystallographic analysis using X-ray diffraction (XRD). Fig. 2 shows the results of this XRD in the as-deposited FePt and FePtSn thin films. Only the facecentered cubic (fcc) (200) peak was observed in both films, suggesting that the $\mathrm{MgO}$ single-crystal substrate is effective in preventing fcc(111) grain growth. It is worth noting that the $\mathrm{fcc}(200)$ peak of the FePtSn thin-film was shifted about $-0.153^{\circ}$ from that of the FePt film, indicating that $\mathrm{Sn}$ causes an expansion of the lattice constant of the FePt alloy. From a crystallographic analysis of the XRD results, we find that the lattice constant of the FePtSn film was expanded by about $1.1 \%$ compared with the lattice constant of the FePt film.

The XRD results for the FePt and FePtSn films annealed at $600^{\circ} \mathrm{C}$ are shown in Fig. 3. After the annealing at $600^{\circ} \mathrm{C}$, the $L 1_{0}(001)$ peak was observed, and the $L 1_{0}(200)$ and $L 1_{0}(002)$ peaks overlapped in both films. Comparing the $I_{001} / I_{\mathrm{MgO}}$ and $I_{002} / I_{\mathrm{MgO}}$ intensity ratios of the FePt and FePtSn films, we see that the (001) texture of the FePtSn film is more developed than that of the FePt film. This developed texture is thought to enhance the coercivity.

In order to quantitatively describe the change in the ordering degree of the FePt phase in the films annealed at $600^{\circ} \mathrm{C}$, we introduce the ordering parameter $\mathrm{S}$. The relation- 


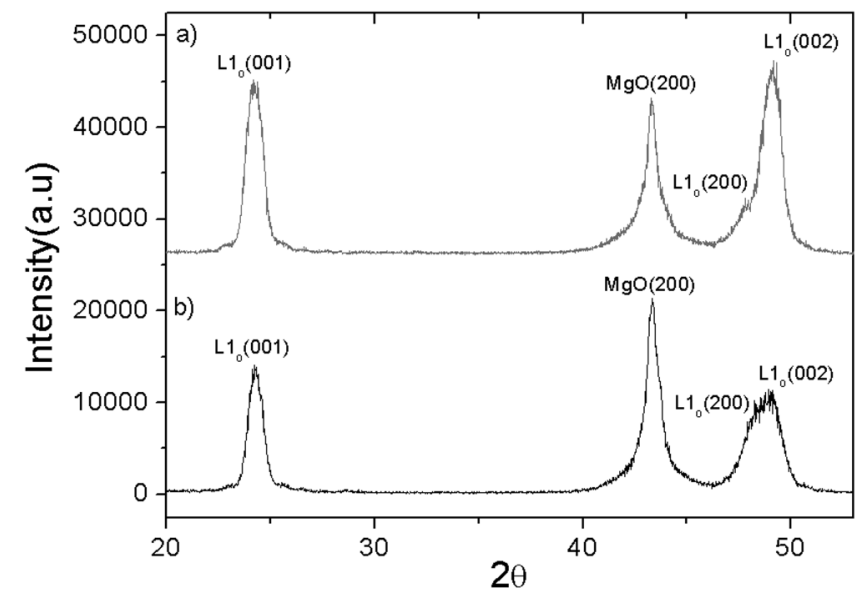

Fig. 3. X-ray diffraction of a) FePtSn thin film and b) FePt thin film after annealing at $600^{\circ} \mathrm{C}$.

ship between $\mathrm{S}$ and c/a can be expressed as follows:

$$
S^{2}=\frac{1-(c / a)}{1-(c / a)_{S_{f}}}
$$

where (c/a)sf $=0.961$ is the axial ratio for the fully ordered phase and (c/a) is the axial ratio for the partially ordered phase and can be evaluated from the superlattice and fundamental peaks in the XRD patterns. At an annealing temperature of $600^{\circ} \mathrm{C}$, the ordering parameter $\mathrm{S}$ for FePtSn (1.3 at.\%) is 0.7298, whereas the $\mathrm{S}$ for FePt is 0.6204 , indicating that $\mathrm{Sn}$ is effective in reducing the ordering temperature of $\mathrm{FePt}$ alloy.

As mentioned above, the lattice constant of the asdeposited FePtSn film was larger than that of the as-deposited FePt film, indicating that $\mathrm{Sn}$ is being incorporated into the lattice of FePt and forming a solid solution. When annealed at temperatures above $500^{\circ} \mathrm{C}$, both films transformed from the disordered state to the ordered one. However, the (001) and (002) peaks were at the same position in the FePt and FePtSn films, suggesting that the lattice constants of the two films coincide at the annealing temperature of $600^{\circ} \mathrm{C}$.

This observation suggests that the effect of adding Sn to the FePt film is to introduce a large lattice strain primarily in the as-deposited states. The added Sn atoms can be supersaturated in the as-deposited FePt phase, and they can cause a large lattice strain, which in turn provides a strong driving force for the transformation to the ordered phase. The solubility of $\mathrm{Sn}$ in ordered FePt is comparatively small, and thus excess Sn atoms are expected to precipitate out from the cubic phase during annealing processes higher than $500^{\circ} \mathrm{C}$.

To investigate the distribution of Sn after the annealing process, we performed a high-resolution TEM analysis on

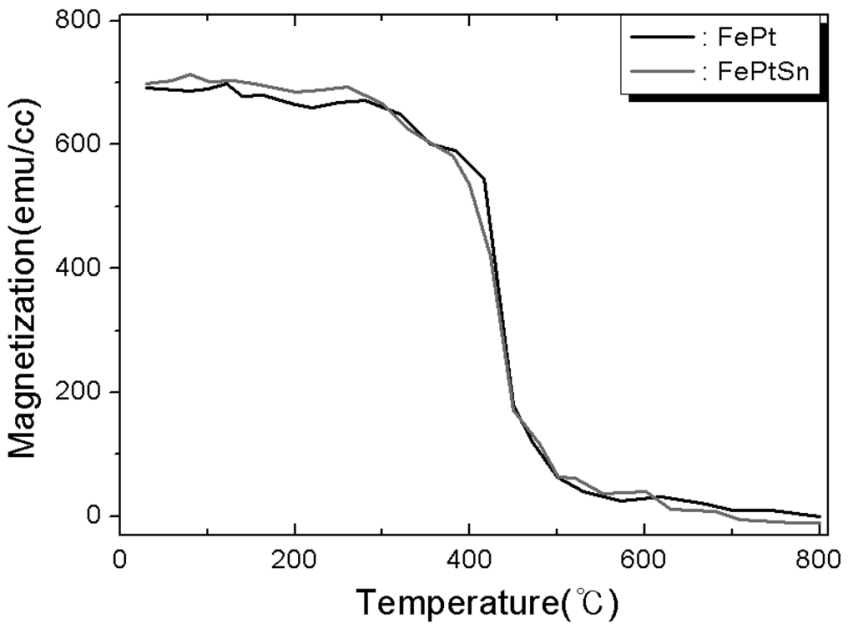

Fig. 4. Temperature-dependent magnetization of the FePt and FePtSn thin films.

the FePtSn film before and after annealing. No evidence of a grain boundary phase or a precipitate was found, which leads us to expect that the excess $\mathrm{Sn}$ atoms in the annealed FePtSn film may have segregated to the surface or to interfacial regions of the film because of the reduction of the surface energy [10].

Fig. 4 shows the temperature-dependent magnetization of both films. If the addition of $\mathrm{Sn}$ formed a solid solution in the FePt alloy, then the $T_{\mathrm{C}}$ of the FePtSn film would be different from that of the FePt film. We can see from Fig. 4, however, that the $T_{\mathrm{C}}$ of both films is almost the same, suggesting that the added $\mathrm{Sn}$ atoms must have diffused out from the FePt lattice.

The enhancement of the coercivity of FePt with the addition of Sn can be attributed to the lowering of the activation energy of the order-disorder transformation in the FePt system. High internal strain and lattice defects caused by the formation of a supersaturated solid solution of FePtSn are thought to be the major factors contributing to the faster kinetics of the phase transformation. This behavior is confirmed by the rapid increase in the coercivity of the FePtSn film at annealing temperatures lower than that of the FePt film.

\section{Conclusions}

In this work, we studies the effects of the addition of Sn on the magnetic properties and the microstructure of FePt thin films. The coercivity of FePtSn film was approximately 1000 Oe greater than that of the FePt film for an annealing temperature of $600^{\circ} \mathrm{C}$. Our results show that the Sn addition was effective in reducing the ordering temperature of $L 1_{0} \mathrm{FePt}$ alloy. Moreover, we demonstrated that the lattice constant of the as-deposited FePtSn is expand- 
ed because of to the induced internal stress originating from the supersaturated $\mathrm{Sn}$ atoms in the FePt lattice. These supersaturated $\mathrm{Sn}$ atoms reduce the activation energy for the $L 1_{0}$ ordering of FePt alloy. The excess Sn originating from the solid-solubility difference between the disordered and ordered states of the FePt alloy diffused out to the surface and to interfaces in order to minimize the surface energy [11].

\section{Acknowledgment}

This research was supported by a grant from the Fundamental R\&D Program for Core Technology of Materials funded by the Ministry of Commerce, Industry and Energy, Republic of Korea.

\section{References}

[1] Shouheng Sun, C. B. Murray, Dieter Weller, Liesl Folks and Andreas Moser, Science 287, 1989-1992 (2000).

[2] Jiao-Ming Qiu and Jian-Ping Wang, Advanced Materials
19, 1703-1706 (2007).

[3] Yingfan, J. S. Chen, and J. P. Wang, Appl. Phys. Lett. 80, 3325-3327 (2002).

[4] W. K. Shen, J. H. Judy, and Jian-Ping Wang, J. Appl. Phys. 97, 10H301 (2007).

[5] T. Shima, T. Moriguchi, S. Mitani, and K. Takanashi, Appl. Phys. Lett. 80, 288-290 (2002).

[6] Yu-Nu Hsu, Sangki Jeong, and David E. Laughlin, J. Appl. Phys. 89, 7068-7070 (2001).

[7] C. C. Chiang, Chih-Huang Lai, and Y. C. Wu, Appl. Phys. Lett. 88, 152508 (2006).

[8] Tomoyuki Maeda, Tadashi Kai, Akira Kikitsu, Toshihiki Nagase, and Jun-ichi Akiyama, Appl. Phys. Lett. 80, 2147-2149 (2002).

[9] C. L. Platt, K. W. Wierman, E. B. Svedberg, R. van de Veerdonk, A. g. Roy, D. E. Laughlin and K. K. Howard, J. Appl. Phys. 92, 6104-6109 (2002).

[10] O. Kitakami, K. Oikawa, H. Daimon, K. Fukamichi, and Y. Shimada, Appl. Phys. Lett. 78, 1104-1106.

[11] D. W. Chun, S. M. Kim, G. H. Kim, and W. Y. Jeung, J. Appl. Phys. (accepted). 